\title{
On robustness of finite-time stability of homogeneous affine nonlinear systems and cascade interconnections ${ }^{*}$
}

\author{
Youness Braidiz $^{\mathrm{a}, \mathrm{b}}$, Denis Efimov ${ }^{\mathrm{b}, \mathrm{c}}$, Andrey Polyakov ${ }^{\mathrm{b}, \mathrm{c}}$, Wilfrid Perruquetti ${ }^{\mathrm{a}}$
}

\author{
ARTICLE HISTORY \\ Compiled September 9, 2020
}

\begin{abstract}
This paper investigates robustness of finite-time stability property for a homogeneous nonlinear dynamical system with sufficiently small affine inputs. In addition, robust stability conditions are presented for the systems admitting homogeneous approximations at the origin or at infinity. The effects of additional stable unmodeled dynamics in the input channel on robust stability are investigated. The utility of the obtained results is illustrated via robustness analysis of homogeneous observer with time-varying gains.
\end{abstract}

\section{KEYWORDS}

Homogeneous systems; robustness; finite-time stability.

\section{Introduction}

The problem of robustness analysis of nonlinear control systems is at the center of attention of researchers for several decades (Arcak \& Kokotović, 2001; Chaillet, Angeli, \& Ito, 2014a, 2014b; Freeman \& Kokotovic, 2008; Jiang, Teel, \& Praly, 1994; Sontag, 1989, 1998), etc.

One of the most popular robust stability properties, which was introduced in (Sontag, 1989), is the concept of input-to-state stability (ISS). This framework has become indispensable for various branches of nonlinear control theory, such as design of nonlinear observers (Arcak \& Kokotović, 2001), robust stabilization of nonlinear systems (Freeman \& Kokotovic, 2008), etc. However, sometimes it is impossible to ensure the ISS behavior of a closed loop system globally, and its local variant (LISS) is frequently used. Moreover, another relaxation of the ISS concept, known as integral input-to-state stability (iISS), has been proposed in (Sontag, 1998). The following interpretation of these notions is possible: while the state of an ISS system is small if inputs are small, the state of an iISS system is small if inputs have a finite energy. Moreover, every ISS system is necessarily iISS, but the converse is not true. It has been shown that ISS property (resp., iISS, LISS) are equivalent to the existence of a smooth ISS (resp.,

\footnotetext{
${ }^{*}$ This work was partially supported by ANR 15 CE23 0007 (Project Finite4SoS), by the Government of Russian Federation (Grant 08-08) and by the Ministry of Science and Higher Education of Russian Federation, passport of goszadanie no. 2019-0898.

${ }^{a}$ Ecole Centrale, Univ. Lille, CNRS, UMR 9189 - CRIStAL, F-59000 Lille, France.

bInria, Univ. Lille, CNRS, UMR 9189 - CRIStAL, F-59000 Lille, France.

${ }^{\mathrm{c}}$ ITMO University, 49 av. Kronverkskiy, 197101 Saint Petersburg, Russia.
} 
iISS, LISS) Lyapunov function (Sontag \& Wang, 1995), which allows this framework to be widely used.

Another related notion, named strong iISS, was introduced in (Chaillet et al., 2014b). This new concept is given as an intermediate property, which offers an interesting compatibility between ISS and the generality of iISS. This concept ensures that the trajectories of any strongly iISS system are globally bounded provided that the input amplitude is less than a specific constant, when it is greater than this constant we recover a conventional iISS performance. The strong iISS property is found useful for analysis of cascade-interconnected systems (Chaillet et al., 2014b).

Following (Braidiz, Efimov, Polyakov, \& Perruquetti, 2019), in this paper we are going to develop these robust stability concepts for a class of homogeneous systems and their interconnections. Many results dealing with analysis and design have been proposed for this kind of models, see (Bacciotti \& Rosier, 2006; Bernuau, Efimov, Perruquetti, \& Polyakov, 2013, 2014; Bernuau, Polyakov, Efimov, \& Perruquetti, 2013b; Bhat \& Bernstein, 2005; Coron, 2007; Efimov \& Perruquetti, 2010; Hahn, 1967; Hahn, Hosenthien, \& Lehnigk, 1963). Such a popularity of homogeneous systems follows their various advantages. And one of them consists in ability of this type of dynamics to converge in finite-time to the origin. The finite-time stability (FTS) was discovered by Erugin 1951, Zubov 1957 and studied by Roxin 1966. It becomes popular in many applications needed an accelerated convergence of the trajectories to the goal reference, and studied by (Bhat \& Bernstein, 2000, 2005; Korobov, 1979; Moulay \& Perruquetti, 2006; Roxin, 1966).

The goal of this work is to investigate the robustness of (homogeneous) FTS systems with respect to exogenous (small) inputs by applying the ISS framework and uniform stability concepts. The basic conditions of ISS and iISS for homogeneous systems have been established in (Bernuau, Polyakov, et al., 2013b), and in this work we are going to go beyond with more evolved analysis.

To this end this paper is organized as follows. After introducing definitions of robust stability and generalized homogeneity in Section 2, we will investigate the robustness of homogeneous affine nonlinear systems, and the robustness of a system which admits a homogeneous approximation (that is locally homogeneous) in Section 3. Next, we will also study the robust stability of the interconnected systems with homogeneous subsystems in Section 3. These results are applied in Section 4 to analysis of the estimation error dynamics of an observer introduced in (Perruquetti, Floquet, \& Moulay, 2008).

\section{Preliminaries}

In this section, after notation we will introduce the used in the sequel robust stability concepts (subsection 2.2) and the framework of generalized homogeneous systems (subsection 2.3).

\subsection{Notation}

- $\mathbb{R}_{+}=\{x \in \mathbb{R}: x \geq 0\}$, where $\mathbb{R}$ is the set of real numbers; $|\cdot|$ denotes the absolute value in $\mathbb{R}$ and $\|\cdot\|$ denotes the Euclidean norm in $\mathbb{R}^{n}$.

- $S=\left\{x \in \mathbb{R}^{n}:\|x\|=1\right\}$ denotes the unit sphere in $\mathbb{R}^{n}$, and $\|A\|_{\mathcal{M}_{m, n}}=$ $\sup _{x \in S}\|A x\|, A \in \mathcal{M}_{m, n}$, where $\mathcal{M}_{m, n}$ is the set of all $m \times n$-matrices over the $x \in S$ 
field of real numbers, it forms a vector space and $\|\cdot\|_{\mathcal{M}_{m, n}}$ denotes the matrix norm induced by $\|\cdot\|$. When $m=n$ we write $\mathcal{M}_{n}$ instead of $\mathcal{M}_{n, n}$.

- For a (Lebesgue) measurable function $d: \mathbb{R}_{+} \rightarrow \mathbb{R}^{m}$ define the norm $\|d\|_{\left[t_{0}, t_{1}\right)}=$ $\operatorname{ess} \sup _{t \in\left[t_{0}, t_{1}\right)}\|d(t)\|$, then $\|d\|_{\infty}=\|d\|_{[0,+\infty)}$ and the set of $d$ with the property $\|d\|_{\infty}<+\infty$ we further denote as $\mathcal{L}_{\infty}$ (the set of essentially bounded measurable functions).

- A continuous function $\alpha: \mathbb{R}_{+} \rightarrow \mathbb{R}_{+}$belongs to the class $\mathcal{K}$ if $\alpha(0)=0$ and the function is strictly increasing. The function $\alpha: \mathbb{R}_{+} \rightarrow \mathbb{R}_{+}$belongs to the class $\mathcal{K}_{\infty}$ if $\alpha \in \mathcal{K}$ and it is increasing to infinity. A continuous function $\beta$ : $\mathbb{R}_{+} \times \mathbb{R}_{+} \rightarrow \mathbb{R}_{+}$belongs to the class $\mathcal{K} \mathcal{L}$ if $\beta(\cdot, t) \in \mathcal{K}_{\infty}$ for each fixed $t \in \mathbb{R}_{+}$; it is a decreasing function in the second argument and $\lim _{t \rightarrow+\infty} \beta(s, t)=0$ for each fixed $s \in \mathbb{R}_{+} \backslash\{0\}$. A continuous function $\beta: \mathbb{R}_{+} \times \mathbb{R}_{+} \rightarrow \mathbb{R}_{+}$belongs to the class $\mathcal{G} \mathcal{K} \mathcal{L}$ if $\beta(\cdot, 0) \in \mathcal{K}_{\infty}, \beta(s, \cdot)$ is a decreasing function for any fixed $s \in \mathbb{R}_{+} \backslash\{0\}$ and $\beta(s, \tau)=0, \forall \tau \geq T$ for each fixed $s \in \mathbb{R}_{+}$for some $0 \leq T<+\infty$.

- The notation $D V(x)=\left(\frac{\partial V}{\partial x_{1}}(x), \cdots, \frac{\partial V}{\partial x_{n}}(x)\right)^{T}$ stands for the first derivative of a continuously differentiable function $V$ at point $x$.

- The notation $\langle D V(x), f(x)\rangle=\sum_{i=1}^{n} \frac{\partial V}{\partial x_{i}}(x) f_{i}(x)$ stands for the directional derivative of a continuously differentiable function $V$ with respect to the vector field $f$ evaluated at point $x \in \mathbb{R}^{n}$.

- $\mathcal{C}^{p}\left(\mathbb{R}^{n}, \mathbb{R}\right)$ denotes the space of functions $f: \mathbb{R}^{n} \rightarrow \mathbb{R}$ which have $p$ continuous derivatives and $\mathcal{C}^{\infty}\left(\mathbb{R}^{n}, \mathbb{R}\right)$ denotes the space of smooth functions. We denote by $\mathcal{C} \mathcal{L}^{p}\left(\mathbb{R}^{n}, \mathbb{R}\right)$ a subset of $\mathcal{C}^{0}\left(\mathbb{R}^{n}, \mathbb{R}\right)$ that is $\mathcal{C}^{p}\left(\mathbb{R}^{n} \backslash\{0\}, \mathbb{R}\right)$.

\subsection{Stability properties}

In this subsection we give some definitions of robust stability (for more details see (Bernuau, Polyakov, et al., 2013b)) and FTS, which will be investigated for systems with disturbances:

$$
\dot{x}(t)=f(x(t), \delta(t)), \quad t \geq 0,
$$

where $x(t) \in \mathbb{R}^{n}$ is the state and $\delta(t) \in \mathbb{R}^{m}$ is the external input, $\delta \in \mathcal{L}_{\infty}$. The vector field $f: \mathbb{R}^{n} \times \mathbb{R}^{m} \rightarrow \mathbb{R}^{n}$ with $f(0,0)=0$ is assumed to be such that the solutions of (1) exist and they are uniquely defined at least locally in forward time. For an initial condition $x_{0} \in \mathbb{R}^{n}$ and input $\delta \in \mathcal{L}_{\infty}$, define the corresponding solution by $x\left(t, x_{0}, \delta\right)$ for any $t \geq 0$ for which the solution exists.

\subsubsection{Finite-time stability}

First, let us define the basic stability notions and their Lyapunov characterizations. Let $\mathcal{V} \subset \mathbb{R}^{n}$ be a nonempty open neighborhood of the origin.

Definition 2.1. (Bernuau et al., 2014; Bhat \& Bernstein, 2000) For $\delta=0$ the origin of the system (1) is said to be:

1. Lyapunov stable (LS) if for all $x_{0} \in \mathcal{V}$ the solutions are defined for all $t \geq 0$, and there exists a function $\alpha \in \mathcal{K}$ such that for all $x_{0} \in \mathcal{V}$ we have $\left\|x\left(t, x_{0}, 0\right)\right\| \leq$ $\alpha\left(\left\|x_{0}\right\|\right), \forall t \geq 0$

2. Asymptotically stable (AS) if it is LS and $\lim _{t \rightarrow+\infty}\left\|x\left(t, x_{0}, 0\right)\right\|=0$ for all $x_{0} \in \mathcal{V}$; 
3. FTS if it is LS and there exists a function $T: \mathcal{V} \rightarrow \mathbb{R}^{+}$such that $x\left(t, x_{0}, 0\right)=0$ for all $t \geq T\left(x_{0}\right)$ and all $x_{0} \in \mathcal{V}$.

In addition, if $\mathcal{V}=\mathbb{R}^{n}$, then the origin is a globally AS (GAS) or globally FTS (GFTS). If the properties 1),2) or 1),3) are satisfied for a given set of inputs $\delta$ (with $T$ and $\alpha$ independent on $\delta$ ) we say that the system (1) is uniformly AS or FTS, respectively.

The following proposition shows how to investigate FTS by using the Lyapunov theory.

Proposition 2.2. (Moulay \& Perruquetti, 2006) For $\delta=0$ the origin of the system (1) is FTS with a continuous settling-time function at the origin if and only if there exists a nonempty open neighborhood of the origin $\mathcal{V} \subset \mathbb{R}^{n}$, real numbers $c>0,0<$ $\alpha<1$ and a positive definite Lyapunov function $V \in \mathcal{C} \mathcal{L}^{1}\left(\mathcal{V}, \mathbb{R}_{+}\right)$satisfying

$$
\langle D V(x), f(x, 0)\rangle \leq-c[V(x)]^{\alpha}, \forall x \in \mathcal{V} .
$$

\subsubsection{ISS and related notions}

Next, let us introduce robust stability properties, which are investigated in this work.

Definition 2.3. (Hong, Jiang, \& Feng, 2008; Sontag, 1989) The system (1) is said to be

- ISS, if there exist $\beta \in \mathcal{K} \mathcal{L}$ and $\gamma \in \mathcal{K}$ such that for any initial state $x_{0} \in \mathbb{R}^{n}$ and any $\delta \in \mathcal{L}_{\infty}$, the solution $x\left(t, x_{0}, \delta\right)$ exists for all $t \geq 0$ and satisfies

$$
\left\|x\left(t, x_{0}, \delta\right)\right\| \leq \beta\left(\left\|x_{0}\right\|, t\right)+\gamma\left(\|\delta\|_{\infty}\right)
$$

- ISS with respect to small inputs if there exists a constant $R>0$ such that for all $x_{0} \in \mathbb{R}^{n}$, the solution $x\left(t, x_{0}, \delta\right)$ exists for all $t \geq 0$ and satisfies

$$
\|\delta\|_{\infty}<R \Longrightarrow\left\|x\left(t, x_{0}, \delta\right)\right\| \leq \beta\left(\left\|x_{0}\right\|, t\right)+\gamma\left(\|\delta\|_{\infty}\right) .
$$

If ISS or ISS with respect to small inputs property holds with $\beta \in \mathcal{G K \mathcal { L }}$, then (1) is called finite-time ISS (FTISS) or FTISS with respect to small inputs, respectively.

If additionally (2) holds only for $x_{0} \in \mathcal{V}$, where $\mathcal{V}$ is a neighborhood of the origin in $\mathbb{R}^{n}$, then the previous properties are called local.

Definition 2.4. (Sontag, 1998) The system (1) is called iISS, if there are some functions $\alpha \in \mathcal{K}_{\infty}, \gamma \in \mathcal{K}$ and $\beta \in \mathcal{K} \mathcal{L}$ such that for any $x_{0} \in \mathbb{R}^{n}$ and $\delta \in \mathcal{L}_{\infty}$ the estimate holds:

$$
\alpha\left(\left\|x\left(t, x_{0}, \delta\right)\right\|\right) \leq \beta\left(\left\|x_{0}\right\|, t\right)+\int_{0}^{t} \gamma(\|\delta(s)\|) d s, \forall t>0 .
$$

If $\beta \in \mathcal{G K} \mathcal{L}$, we say that the system (1) is finite-time iISS (FTiISS).

Definition 2.5. (Chaillet et al., 2014a, 2014b) The system (1) is said to be strongly iISS if it is both iISS and ISS with respect to small inputs. 
Definition 2.6. The system (1) is said to be finite-time strongly iISS if it is both FTiISS and FTISS with respect to small inputs.

If in the above definitions we replace the distance to the origin $\|x\|$ with the distance to a compact set, then we can recover the respective stability characterizations with respect to this set.

All these stability properties can be verified by looking for existence of a corresponding Lyapunov function:

Definition 2.7. (Bernuau et al., 2014; Sontag \& Wang, 1995) A $V \in \mathcal{C}^{1}\left(\mathbb{R}^{n}, \mathbb{R}_{+}\right)$is called

- an ISS Lyapunov function if for all $x \in \mathbb{R}^{n}, \delta \in \mathbb{R}^{m}$ and some $\alpha_{1}, \alpha_{2}, \alpha_{3} \in \mathcal{K}_{\infty}$ and $\gamma \in \mathcal{K}$ :

$$
\begin{gathered}
\alpha_{1}(\|x\|) \leq V(x) \leq \alpha_{2}(\|x\|), \\
\langle D V(x), f(x, \delta)\rangle \leq-\alpha_{3}(\|x\|)+\gamma(\|\delta\|),
\end{gathered}
$$

such a function $V$ is called ISS Lyapunov function with respect to a compact set $\mathcal{A}=\left\{x \in \mathbb{R}^{n}: V(x) \leq A\right\}$ for some $A \geq 0$, if the inequality (5) holds for all $x \in \mathbb{R}^{n} \backslash \mathcal{A}$;

- an iISS Lyapunov function if (4) and (5) hold for a positive definite function $\alpha_{3}: \mathbb{R}_{+} \rightarrow \mathbb{R}_{+}$

- a FTISS (resp., FTiISS) Lyapunov function if (4) and (5) hold and there exists $\varepsilon>0$ such that $\alpha_{3}(\|x\|) \geq c V(x)^{\alpha}$ for all $\|x\| \leq \varepsilon$ with $c>0$ and $0<\alpha<1$;

- a local ISS Lyapunov function if there exists $r>0$ such that the inequalities (4) and (5) hold $\forall x \in \mathbb{R}^{n}$ with $\|x\| \leq r, \forall \delta \in \mathbb{R}^{m}$ with $\|\delta\| \leq r$.

Note that an ISS Lyapunov function can also satisfy instead of (5) an equivalent condition for some $\chi \in \mathcal{K}$ and $\alpha_{3} \in \mathcal{K}_{\infty}$ (see (Sontag \& Wang, 1995)):

$$
\|x\| \geq \chi(\|\delta\|) \Longrightarrow\langle D V(x), f(x, \delta)\rangle \leq-\alpha_{3}(\|x\|) .
$$

Relations between existence of ISS (iISS, FTISS, FTiISS) Lyapunov functions and ISS (iISS, FTISS, FTiISS) properties are presented in the following theorems:

Theorem 2.8. (Angeli, Sontag, 8 Wang, 2000; Sontag 86 Wang, 1995) The following properties are equivalent:

1) The system (1) is ISS (iISS) with $\delta$ as input;

2) There is an ISS (iISS) Lyapunov function $V \in \mathcal{C}^{1}\left(\mathbb{R}^{n}, \mathbb{R}_{+}\right)$.

Theorem 2.9. (Bernuau, Polyakov, Efimov, \& Perruquetti, 2013a) If for the system (1) there exists a (local) FTISS (FTiISS) Lyapunov function, then it is (locally) FTISS (FTiISS).

Note that to check strong iISS property we can unite the Lyapunov characterizations given before for iISS and ISS (the same for finite-time counterparts).

The last theorem in this subsection is about preservation of strong iISS under cascade interconnection: 
Theorem 2.10. (Chaillet et al., 2014b) Assume that the systems $\dot{x}_{1}=f_{1}\left(x_{1}, \delta_{1}\right)$ and $\dot{x}_{2}=f_{2}\left(x_{2}, \delta_{2}\right)\left(f_{1}(0,0)=0, f_{2}(0,0)=0\right)$ are strongly iISS with respect to $\delta_{1}$ and $\delta_{2}$ respectively. Then the cascade

$$
\left\{\begin{array}{l}
\dot{x}_{1}=f_{1}\left(x_{1}, x_{2}\right) \\
\dot{x}_{2}=f_{2}\left(x_{2}, \delta_{2}\right)
\end{array}\right.
$$

is strongly iISS.

\subsection{Generalized homogeneity}

In control theory, homogeneity is a special kind of Lie symmetry, which simplifies qualitative analysis of nonlinear dynamic systems. In particular, it allows local properties (e.g., local stability) to be extended globally using a scaling or dilation of the solutions.

Definition 2.11. A map $\mathbf{d}: \mathbb{R} \rightarrow \mathcal{M}_{n}$ is called a dilation in $\mathbb{R}^{n}$ iff it satisfies

- Group property: $\mathbf{d}(0)=I_{n}, \mathbf{d}(t+s)=\mathbf{d}(t) \mathbf{d}(s), t, s \in \mathbb{R}$;

- Continuity property: $\mathbf{d}$ is continuous, i.e. $\forall t>0, \forall \varepsilon>0, \exists \gamma>0$ : $|s-t|<$ $\gamma \Longrightarrow\|\mathbf{d}(s)-\mathbf{d}(t)\|_{\mathcal{M}_{n}} \leq \varepsilon$

- Limit property: $\lim _{s \rightarrow-\infty}\|\mathbf{d}(s) x\|=0$ and $\lim _{s \rightarrow+\infty}\|\mathbf{d}(s) x\|=+\infty$ uniformly on the unit sphere $S$.

Definition 2.12. The dilation $\mathbf{d}$ is monotone in $\mathbb{R}^{n}$ if $\|\mathbf{d}(s)\|_{\mathcal{M}_{n}}<1, \forall s<0$.

Any dilation in $\mathbb{R}^{n}$ is monotone under proper selection of the weighted Euclidean norm in $\mathbb{R}^{n}$ Polyakov (2018).

Definition 2.13. The function $\|\cdot\|_{\mathbf{d}}: \mathbb{R}^{n} \rightarrow \mathbb{R}_{+}$is called the canonical homogeneous norm $\|x\|_{\mathbf{d}}=e^{s_{x}}$, where $s_{x} \in \mathbb{R}:\left\|\mathbf{d}\left(-s_{x}\right) x\right\|=1$.

In Polyakov (2018) it is shown that the canonical homogeneous norm is singlevalued and continuous provided that the dilation is monotone. Obviously, it has the properties $\lim _{x \rightarrow 0}\|x\|_{\mathbf{d}}=0$ and $\|\mathbf{d}(s) x\|_{\mathbf{d}}=e^{s}\|x\|_{\mathbf{d}}>0$ for all $x \in \mathbb{R}^{n} \backslash\{0\}$ and all $s \in \mathbb{R}$, in addition

$$
\|x\|_{\mathbf{d}}=1 \Leftrightarrow\|x\|=1 .
$$

Definition 2.14. A vector field $f: \mathbb{R}^{n} \rightarrow \mathbb{R}^{n}$ (resp., a function $h: \mathbb{R}^{n} \rightarrow \mathbb{R}$ ) is said to be $\mathbf{d}$-homogeneous of degree $\nu \in \mathbb{R}$ (resp., $\mu \in \mathbb{R}$ ) if for all $s \in \mathbb{R}$ and all $x \in \mathbb{R}^{n}$ we have $e^{-\nu s} \mathbf{d}(-s) f(\mathbf{d}(s) x)=f(x)$, (resp., $\left.e^{-\mu s} h(\mathbf{d}(s))=h(x)\right)$. We say that a system $\dot{x}=f(x)$ is $\mathbf{d}$-homogeneous if $f$ is $\mathbf{d}$-homogeneous.

Any linear vector field is $\mathbf{d}$-homogeneous of zero degree with $\mathbf{d}(s)=e^{s} I$. Homogeneity property was introduced also for Banach or Hilbert spaces, and it is given by a group of dilations (Polyakov, Coron, \& Rosier, 2018; Polyakov, Efimov, Fridman, \& Perruquetti, 2016).

The following lemma provides a useful comparison between homogeneous functions.

Lemma 2.15. (Bhat \& Bernstein, 2005) Suppose that $V_{1}, V_{2} \in \mathcal{C}^{0}\left(\mathbb{R}^{n}, \mathbb{R}_{+}\right)$are $\boldsymbol{d}$-homogeneous of degrees $l_{1}>0$ and $l_{2}>0$, respectively, and $V_{1}$ is positive definite. 
Then $a_{1}\left[V_{1}(x)\right]^{\frac{l_{2}}{l_{1}}} \leq V_{2}(x) \leq a_{2}\left[V_{1}(x)\right]^{\frac{l_{2}}{l_{1}}}$ for all $x \in \mathbb{R}^{n}$, where $a_{1}=\min _{\left\{z ; V_{1}(z)=1\right\}} V_{2}(z)$ and $a_{2}=\max _{\left\{z ; V_{1}(z)=1\right\}} V_{2}(z)$.

In the following definition we introduce the notion of homogeneous approximations that can be useful for local analysis and design of nonlinear control systems (see (Andrieu, Praly, \& Astolfi, 2008) and (Efimov \& Perruquetti, 2016) for more details).

Definition 2.16. A function (resp., a vector field) $f$ is said to be homogeneous in the 0 -limit with associated triple $\left(\nu_{0}, \mathbf{d}, f_{0}\right)$ if $\lim _{s \rightarrow-\infty} \sup _{x \in S}\left\|e^{-\nu_{0} s} f(\mathbf{d}(s)(x))-f_{0}(x)\right\|=0$ (resp., if $\lim _{s \rightarrow-\infty} \sup _{x \in S}\left\|e^{-\nu_{0} s} \mathbf{d}(-s) f(\mathbf{d}(s)(x))-f_{0}(x)\right\|=0$ ). A function (resp., a vector field) $f$ is said to be homogeneous in the $\infty$-limit with associated triple $\left(\nu_{\infty}, \mathbf{d}, f_{\infty}\right)$ if $\lim _{s \rightarrow+\infty} \sup _{x \in S}\left\|e^{-\nu_{\infty} s} f(\mathbf{d}(s)(x))-f_{\infty}(x)\right\|=0$ (resp., if $\lim _{s \rightarrow+\infty} \sup _{x \in S} \| e^{-\nu_{\infty} s} \mathbf{d}(-s) f(\mathbf{d}(s)(x))-$ $\left.f_{\infty}(x) \|=0\right)$.

Example 2.17. Let $f \quad: \quad\left(x_{1}, x_{2}\right) \in \mathbb{R}^{2} \longmapsto\left(x_{2}, x_{2}^{1.5}+5 x_{2}^{1.7}\right)^{T} \in \mathbb{R}^{2}$. The vector field $f$ is homogeneous in the 0 -limit with associated triple $\left(\nu_{0}, \mathbf{d}_{0}(s), f_{0}(x)\right)=\left(0.5,\left(\begin{array}{ll}e^{0.5 s} & 0 \\ 0 & e^{s}\end{array}\right),\left(x_{2}, x_{2}^{1.5}\right)^{T}\right)$ and in $\infty$-limit with the triple $\left.\left(\nu_{\infty}, \mathbf{d}_{\infty}(s), f_{\infty}(x)\right)\right)=\left(0.7,\left(\begin{array}{ll}e^{0.3 s} & 0 \\ 0 & e^{s}\end{array}\right),\left(x_{2}, 5 x_{2}^{1.7}\right)^{T}\right)$.

The existence of a homogeneous Lyapunov function for a GAS homogeneous system was provided in Rosier (1992); Zubov (1958 (in Russian)) by using weighted dilation. The proof for any other kind of dilation is literally the same:

Theorem 2.18. (Rosier, 1992) For $\delta=0$, assume that the system (1) is $\boldsymbol{d}$ homogeneous with degree $\nu$ and the origin is GAS. Then there exists a d-homogeneous positive definite Lyapunov function $V \in \mathcal{C}^{\infty}\left(\mathbb{R}^{n} \backslash\{0\}, \mathbb{R}_{+}\right) \cap \mathcal{C}^{0}\left(\mathbb{R}^{n}, \mathbb{R}_{+}\right)$, with degree of homogeneity $k>\max \{-\nu, 0\}$, such that

$$
\langle D V(x), f(x, 0)\rangle<0, \forall x \neq 0 .
$$

\section{Robustness analysis of a homogeneous system with respect to multiplicative perturbation}

In this section, first, we will consider stable homogeneous systems, which are affine in the external perturbations, and establish the conditions of uniform (robust) stability. Next, we will analyze locally homogeneous dynamics by looking for similar properties. And finally, a cascade connection will be investigated.

\subsection{Robustness of FTS homogeneous systems}

In this subsection we will study the system (1) satisfying the following hypotheses:

$\mathcal{H}_{1}: f(x, \delta)=f_{1}(x)+f_{2}(x) \delta, \delta \in \mathbb{R}^{m}$.

$\mathcal{H}_{2}: f_{1}: \mathbb{R}^{n} \rightarrow \mathbb{R}^{n}$ and $f_{2}: \mathbb{R}^{n} \rightarrow \mathcal{M}_{n, m}$ are continuous and d-homogeneous with degree of homogeneity $\nu \in \mathbb{R}$ for a monotone dilation $\mathbf{d}$. 
$\mathcal{H}_{3}$ : The system $\dot{x}=f(x, 0)$ is GAS.

Assumptions $\mathcal{H}_{1}$ and $\mathcal{H}_{2}$ imply that the function $f$ is also d-homogeneous with degree of homogeneity $\nu$ for any constant $\delta$.

Theorem 3.1. Assume that the system (1) satisfies assumptions $\mathcal{H}_{1}, \mathcal{H}_{2}$ and $\mathcal{H}_{3}$.

- If $\nu \in \mathbb{R}$, then (1) is uniformly GAS for small inputs.

- If $\nu<0$, then (1) is

- uniformly GFTS for small inputs,

- finite-time strongly iISS (in the sense of Definition 2.6).

Proof. Step I: In this step we will prove the uniform GAS of (1) for any $\nu \in \mathbb{R}$ and small inputs $\delta$. We assume that the system $\dot{x}=f(x, 0)$ is GAS and $\mathbf{d}$ homogeneous. Then, Theorem 2.18 implies that there exists a d-homogeneous positive definite Lyapunov function $V \in \mathcal{C}^{\infty}\left(\mathbb{R}^{n} \backslash\{0\}, \mathbb{R}_{+}\right) \cap \mathcal{C}^{0}\left(\mathbb{R}^{n}, \mathbb{R}_{+}\right)$with degree of homogeneity $k>\max \{-\nu, 0\}$, such that $(7)$ holds. Using Lemma 2.15 with $V_{1}(x)=\|x\|_{\mathbf{d}}$ and $V_{2}(x)=\langle D V(x) . f(x, 0)\rangle$, we get

$$
\langle D V(x), f(x, 0)\rangle \leq-a\|x\|_{\mathbf{d}}^{k+\nu}, \forall x \in \mathbb{R}^{n},
$$

where $a=\min _{\|y\|=1}[-\langle D V(y), f(y, 0)\rangle]$. Let $x \in \mathbb{R}^{n}$ and $y \in S$ be such that $y=$ $\mathbf{d}\left(-\ln \left(\|x\|_{\mathbf{d}}\right)\right) x$, then

$$
\begin{aligned}
& \left\langle D V\left(\mathbf{d}\left(\ln \left(\|x\|_{\mathbf{d}}\right)\right) y\right), f_{2}\left(\mathbf{d}\left(\ln \left(\|x\|_{\mathbf{d}}\right)\right) y\right) \delta\right\rangle \\
& =\left\langle e^{k \ln \left(\|x\|_{\mathbf{d}}\right)} \mathbf{d}\left(-\ln \left(\|x\|_{\mathbf{d}}\right)\right) D V(y), e^{\nu \ln \left(\|x\|_{\mathbf{d}}\right)} \mathbf{d}\left(\ln \left(\|x\|_{\mathbf{d}}\right)\right) f_{2}(y) \delta\right\rangle, \\
& =\|x\|_{\mathbf{d}}^{\nu+k}\left\langle D V(y), f_{2}(y) \delta\right\rangle
\end{aligned}
$$

by using Cauchy-Schwarz inequality and (8), one gets

$$
\begin{aligned}
\langle D V(x), f(x, \delta)\rangle & =\langle D V(x), f(x, 0)\rangle+\left\langle D V\left(\mathbf{d}\left(\ln \left(\|x\|_{\mathbf{d}}\right)\right) y\right), f_{2}\left(\mathbf{d}\left(\ln \left(\|x\|_{\mathbf{d}}\right)\right) y\right) \delta\right\rangle \\
& =\langle D V(x), f(x, 0)\rangle+\|x\|_{\mathbf{d}}^{k+\nu}\left\langle D V(y), f_{2}(y) \delta\right\rangle \\
\leq & -a\|x\|_{\mathbf{d}}^{k+\nu}+\|x\|_{\mathbf{d}}^{k+\nu}\left\langle f_{2}(y)^{T} D V(y), \delta\right\rangle \\
\leq & -a\|x\|_{\mathbf{d}}^{k+\nu}+c\|x\|_{\mathbf{d}}^{k+\nu}\|\delta\|,
\end{aligned}
$$

with $c=\sup _{\|y\|=1}\left\|f_{2}(y)^{T} D V(y)\right\|$

For $\|\delta\| \leq \frac{a}{2 c}$, we have $\langle D V(x), f(x, \delta)\rangle \leq-\frac{a}{2}\|x\|_{\mathbf{d}}^{k+\nu}$, which implies that the system (1) with the assumptions $\mathcal{H}_{1}, \mathcal{H}_{2}$ and $\mathcal{H}_{3}$ is uniformly GAS for small inputs $\delta$ and for every $\nu \in \mathbb{R}$.

Step II: $\nu<0$

(1) Recall that if $\|\delta\| \leq \frac{a}{2 c}$, then $\dot{V}(x(t))=\langle D V(x(t)), f(x(t), \delta(t))\rangle \leq-\frac{a}{2}\|x(t)\|_{\mathbf{d}}^{k+\nu}$. Using the homogeneity of $V$, there exist $v_{1}=\min _{\|x\|_{\mathbf{d}=1}} V(x)$, and $v_{2}=\max _{\|x\|_{\mathbf{d}}=1} V(x)$ such that

$$
v_{1}\|x\|_{\mathbf{d}}^{k} \leq V(x) \leq v_{2}\|x\|_{\mathbf{d}}^{k} .
$$

Consequently, $\dot{V}(x)=\langle D V(x), f(x, \delta)\rangle \leq-\frac{a}{2 v_{2}^{\alpha}} V(x)^{\alpha}$, with $0<\alpha=\frac{k+\nu}{k}<1$, for all $x \in \mathbb{R}^{n}$. This inequality and Proposition 2.2 imply the finite-time rate 
of convergence to the origin globally and uniformly with respect to such small inputs $\delta$.

(2) Let the function $W$ be defined by:

$$
W(x)=\ln [1+V(x)] .
$$

It is continuously differentiable, positive definite and radially unbounded, which means that it is a Lyapunov function for (1) when $\delta=0$. The conditions $k+\nu>0$ and $\nu<0$ give

$$
\|x\|_{\mathbf{d}}^{k+\nu} \leq v\left(\|x\|_{\mathbf{d}}\right), \text { with } v(s)=\left\{\begin{array}{ll}
1, & \text { if } \quad s \leq 1 \\
s^{k}, & \text { if } \quad s \geq 1
\end{array} .\right.
$$

This implies that if $\|x\|_{\mathbf{d}} \leq 1$ then $\frac{\|x\|_{\mathbf{d}}^{k+\nu}}{1+V(x)} \leq \frac{v\left(\|x\|_{\mathbf{d}}\right)}{1+V(x)} \leq 1$. If $\|x\|_{\mathbf{d}} \geq 1$, then $\frac{\|x\|_{\mathrm{d}}^{k+\nu}}{1+V(x)} \leq \frac{\|x\|_{\mathrm{d}}^{k}}{1+v_{1}\|x\|_{\mathrm{d}}^{k}} \leq \frac{1}{v_{1}}$ using (10). Therefore,

$$
\frac{\|x\|_{\mathbf{d}}^{k+\nu}}{1+V(x)} \leq \max \left\{1, \frac{1}{v_{1}}\right\}=v_{3}
$$

Combining the properties (9) and (11) we obtain:

$$
\begin{aligned}
\langle D W(x), f(x, \delta)\rangle & \leq-a \frac{\|x\|_{\mathbf{d}}^{k+\nu}}{1+V(x)}+v_{3}\|\delta\| \\
& \leq-a \frac{\|x\|_{\mathbf{d}}^{k+\nu}}{1+v_{2}\|x\|_{\mathbf{d}}}+v_{3}\|\delta\|, \\
& \leq-\alpha_{3}\left(\|x\|_{\mathbf{d}}\right)+v_{3}\|\delta\|
\end{aligned}
$$

where $\alpha_{3}(s)=a \frac{s^{k+\nu}}{1+v_{2} s}$ is a positive definite function. This implies the iISS property for the system (1) (Definition 2.7). Recall that $e^{W}-1 \geq W$ for any $W \geq 0$, select $\varepsilon>0$, then

$$
\begin{aligned}
\|x\|_{\mathbf{d}} \leq \varepsilon & \Longrightarrow a \frac{\|x\|_{\mathbf{d}}^{k+\nu}}{1+v_{2} \varepsilon^{k}} \leq a \frac{\|x\|_{\mathbf{d}}^{k+\nu}}{1+V(x)} \\
& \Longrightarrow\langle D W(x), f(x, \delta)\rangle \leq-a \frac{\|x\|_{\mathbf{d}}^{k+\nu}}{1+v_{2} \varepsilon^{k}}+v_{3}\|\delta\| \\
& \leq-C V(x)^{\frac{k+\nu}{k}}+v_{3}\|\delta\| \\
& \leq-C W(x)^{\alpha}+v_{3}\|\delta\|,
\end{aligned}
$$

with $C=\frac{a}{v_{2}^{\frac{k+\nu}{k}}\left(1+v_{2} \varepsilon^{k}\right)}$. The fact that $0<\alpha=\frac{k+\nu}{k}<1$ and Theorem 2.9 imply that the system (1) is finite-time iISS. Combining this result with the uniform GFTS for small inputs proven previously (which is stronger than FTISS for small inputs) we substantiates that the system (1) is finite-time strongly iISS.

The theorem is proven.

To conclude analysis of the system (1) given in this subsection, ISS and FTISS conditions are formulated for generalized homogeneous systems with exogenous disturbances (extending the result of (Bernuau, Polyakov, et al., 2013b)). These results will be used in the last subsection to study the interconnected homogeneous systems with different degrees of homogeneity. 
Theorem 3.2. Consider the system (1) with $f \in \mathcal{C}^{0}\left(\mathbb{R}^{n+m}, \mathbb{R}^{n}\right)$, let $e^{-\mu s} \boldsymbol{d}_{x}(-s) f\left(\boldsymbol{d}_{x}(s) x, \boldsymbol{d}_{\delta}(s) \delta\right)=f(x, \delta), \forall s \in \mathbb{R}, \forall x \in \mathbb{R}^{n}$ and $\forall \delta \in \mathbb{R}^{m}$, where $\boldsymbol{d}_{x}$ and $\boldsymbol{d}_{\delta}$ are linear dilations, and the system (1) be GAS for $\delta=0$. Then, the system (1) is ISS for all $\mu \in \mathbb{R}$, and FTISS if $\mu<0$.

Proof. Since the system $\dot{x}=f(x, 0)$ is GAS and homogeneous, there exists a homogeneous Lyapunov function $V$ such that the inequality (8) is satisfied, where $k>$ $\max \{-\mu, 0\}$ is the degree of homogeneity of $V$ and $a=\inf _{\|x\|_{\mathbf{d}_{x}=1}}[-\langle D V(x), f(x, 0)\rangle]$.

Let $s=-\ln \left(\|x\|_{\mathbf{d}_{x}}\right)$ and $\tilde{x}=\mathbf{d}_{x}(s) x$, using the inequality (8), we get

$$
\langle D V(x), f(x, \delta)\rangle \leq\|x\|_{\mathbf{d}_{x}}^{\mu+k}\left(-a+b \sup _{\|\tilde{x}\|_{\mathbf{d}_{x}=1}}\left\|f\left(\tilde{x}, \mathbf{d}_{\delta}(s) \delta\right)-f(\tilde{x}, 0)\right\|\right)
$$

with $b=\sup _{\|x\|_{\mathbf{d}_{x}=1}}\|D V(x)\|$. The continuity of the function $f$ implies that there exists a constant $c>0$ such that

$$
\begin{aligned}
\|x\|_{\mathbf{d}_{x}} \geq c^{-1}\|\delta\|_{\mathbf{d}_{\delta}} \Longleftrightarrow & \left\|\mathbf{d}_{\delta}(s) \delta\right\|_{\mathbf{d}_{\delta}} \leq c \Longrightarrow \sup _{\|\tilde{x}\|_{\mathbf{d}_{x}=1}}\left\|f\left(\tilde{x}, \mathbf{d}_{\delta}(s) \delta\right)-f(\tilde{x}, 0)\right\| \leq \frac{a}{2 b} \\
& \Longrightarrow\langle D V(x), f(x, \delta)\rangle \leq-\frac{a}{2}\|x\|_{\mathbf{d}_{x}}^{\mu+k}
\end{aligned}
$$

which due to (6) proves the ISS property of the system (1). If $\mu<0$, by applying the arguments as before we deduce that the system (1) is FTISS.

\subsection{Robustness of locally homogeneous systems}

In this subsection, the robust stability will be investigated for a system, which admits a homogeneous approximation either at 0 or at $\infty$, and whose approximation dynamics satisfy the assumptions $\mathcal{H}_{1}, \mathcal{H}_{2}$ and $\mathcal{H}_{3}$.

Theorem 3.3. Assume that $\forall \delta \in \mathbb{R}^{m}, f(\cdot, \delta)$ is homogeneous in 0-limit with associated triple $\left(\nu_{0}, \boldsymbol{d}, f_{0}(\cdot, \delta)\right)$ where $f_{0}$ satisfies the assumptions $\mathcal{H}_{1}, \mathcal{H}_{2}$ and $\mathcal{H}_{3}$. Then,

(1) if $\nu_{0} \in \mathbb{R}$, the system (1) is uniformly $A S$ for small inputs;

(2) if $\nu_{0}<0$, the system (1) is uniformly FTS for small inputs.

Proof. (1) We assume that the function $f(\cdot, \delta): \mathbb{R}^{n} \rightarrow \mathbb{R}^{n}$ has a homogeneous approximation $f_{0}(\cdot, \delta)$, i.e.

$$
\lim _{s \rightarrow-\infty}\left\|e^{-\nu_{0} s} \mathbf{d}(-s) f(\mathbf{d}(s) x, \delta)-f_{0}(x, \delta)\right\|=0, \forall x \in S, \forall \delta \in \mathbb{R}^{m} .
$$

The function $f_{0}$ satisfies the assumptions $\mathcal{H}_{1}, \mathcal{H}_{2}$ and $\mathcal{H}_{3}$. Similarly to the proof of Theorem 3.1, there exists d-homogeneous Lyapunov function $V$ of degree $k>\max \left\{-\nu_{0}, 0\right\}$ and two positive constant $a$ and $c$ such that

$$
\left\langle D V(x), f_{0}(x, \delta)\right\rangle \leq-a\|x\|_{\mathbf{d}}^{k+\nu_{0}}+c\|x\|_{\mathbf{d}}^{k+\nu_{0}}\|\delta\|, \forall x \in \mathbb{R}^{n}, \forall \delta \in \mathbb{R}^{m} .
$$

Below we will use the coordinate transformation $x=\mathbf{d}(s) y$ with $s=\ln \left(\|x\|_{\mathbf{d}}\right)$, 
which connects any $x \in \mathbb{R}^{n} \backslash\{0\}$ with corresponding point $y \in S$, leading to

$$
\begin{aligned}
\langle D V(x), f(x, \delta)\rangle & =\langle D V(\mathbf{d}(s) y), f(\mathbf{d}(s) y, \delta)\rangle \\
& =\left\langle e^{k s} \mathbf{d}(-s) D V(y), f(\mathbf{d}(s) y, \delta)\right\rangle \\
& =e^{\left(k+\nu_{0}\right) s}\left\langle D V(y), e^{-\nu_{0} s} \mathbf{d}(-s) f(\mathbf{d}(s) y, \delta)\right\rangle \\
& =e^{\left(k+\nu_{0}\right) s}\left\langle D V(y), f_{0}(y, \delta)\right\rangle \\
& +e^{\left(k+\nu_{0}\right) s}\left\langle D V(y),\left(e^{-\nu_{0} s} \mathbf{d}(-s) f(\mathbf{d}(s) y, \delta)-f_{0}(y, \delta)\right)\right\rangle \\
& \leq\|x\|_{\mathbf{d}}^{k+\nu_{0}}(-a+c\|\delta\|) \\
& +\|x\|_{\mathbf{d}}^{k+\nu_{0}}\left\langle D V(y),\left(e^{-\nu_{0} s} \mathbf{d}(-s) f(\mathbf{d}(s) y, \delta)-f_{0}(y, \delta)\right)\right\rangle .
\end{aligned}
$$

It is clear that $s \rightarrow-\infty$ when $\|x\|_{\mathbf{d}} \rightarrow 0$ and the property (12) holds uniformly on $S$, then it implies that we can define an open set containing the origin $\mathcal{V}=$ $\left\{x \in \mathbb{R}^{n}: x=\mathbf{d}(s) y, \forall y \in S, \forall s<\epsilon_{a}\right\}$ such that $\epsilon_{a} \in \mathbb{R}$ satisfies:

$$
\left\langle\frac{\partial V}{\partial y}(y),\left(e^{-\nu_{0} s} \mathbf{d}(-s) f(\mathbf{d}(s) y, \delta)-f_{0}(y, \delta)\right)\right\rangle \leq \frac{a}{2}, \forall y \in S, \forall s<\epsilon_{a} .
$$

Thus,

$$
\langle D V(x), f(x, \delta)\rangle \leq-\frac{a}{2}\|x\|_{\mathbf{d}}^{k+\nu_{0}}+c\|x\|_{\mathbf{d}}^{k+\nu_{0}}\|\delta\|, \forall x \in \mathcal{V}, \forall \delta \in \mathbb{R}^{m} .
$$

Therefore, for $\delta \in \mathbb{R}^{m}$ such that $\|\delta\| \leq \frac{a}{4 c}$ we have

$$
\langle D V(x), f(x, \delta)\rangle \leq-\frac{a}{4}\|x\|_{\mathbf{d}}^{k+\nu_{0}}, \forall x \in \mathcal{V} .
$$

This implies that the system (1) in $\mathcal{V}$ is uniformly AS for small inputs and for any $\nu_{0} \in \mathbb{R}$.

(2) Note that if $\nu_{0}<0$ and the inputs are small (i.e., $\delta \in \mathbb{R}^{m}$, $\|\delta\| \leq \frac{a}{4 c}$ ), then by using (10), one gets

$$
\langle D V(x), f(x, \delta)\rangle \leq-\frac{a}{4}\|x\|_{\mathbf{d}}^{k+\nu_{0}} \leq-\frac{a}{4 v_{2}^{\alpha}} V(x)^{\alpha}, \forall x \in \mathcal{V},
$$

with $0<\alpha=\frac{k+\nu_{0}}{k}<1$. Then, the system preserves a uniform finite-time rate of convergence to the origin locally.

That was necessary to prove.

Clearly, considering homogeneous approximation at 0 leads to a local version of results established in Theorem 3.1. Less intuitive conclusions can be obtained for approximation at $\infty$ :

Theorem 3.4. Assume that $\forall \delta \in \mathbb{R}^{m}, f(\cdot, \delta)$ is homogeneous in $\infty$-limit with respect to the triple $\left(\nu_{\infty}, \boldsymbol{d}, f_{\infty}(\cdot, \delta)\right)$, where the function $f_{\infty}$ satisfies the assumptions $\mathcal{H}_{1}, \mathcal{H}_{2}$ and $\mathcal{H}_{3}$. Then,

(1) if $\nu_{\infty} \in \mathbb{R}$, the system (1) is uniformly GFTS with respect to the set $\mathcal{A}=$ $\left\{x \in \mathbb{R}^{n}:\|x\|_{d} \leq A\right\}$ for some $A>0$ and small inputs;

(2) if $\nu_{\infty}<0$, the set $\mathcal{A}$ is iISS;

(3) if $\nu_{\infty}>0$, the rate of convergence to the set $\mathcal{A}$ is uniform (independent on initial conditions) for small inputs. 
Proof. (1) Repeating the arguments of Theorem 3.3, there is a Lyapunov function $V$ such that

$$
\begin{aligned}
\langle D V(x), f(x, \delta)\rangle & \leq\|x\|_{\mathbf{d}}^{k+\nu_{\infty}}(-a+c\|\delta\|) \\
& +\|x\|_{\mathbf{d}}^{k+\nu_{\infty}}\left\langle D V(y),\left(e^{-\nu_{\infty} s} \mathbf{d}(-s) f(\mathbf{d}(s) y, \delta)-f_{\infty}(y, \delta)\right)\right\rangle .
\end{aligned}
$$

Using the definition of $f_{\infty}$ and the fact that the limit of $\left\langle D V(y),\left(e^{-\nu_{\infty} s} \mathbf{d}(-s) f(\mathbf{d}(s) y, \delta)-f_{\infty}(y, \delta)\right)\right\rangle$ goes to zero when $s$ tends to $+\infty$, we deduce that there exists a positive constant $A$ such that for all $x \in \mathbb{R}^{n}$ and $\forall \delta \in \mathbb{R}^{m}$

$$
\|x\|_{\mathbf{d}}>A,\|\delta\| \leq \frac{a}{4 c} \Longrightarrow\langle D V(x), f(x, \delta)\rangle \leq-\frac{a}{4}\|x\|_{\mathbf{d}}^{k+\nu_{\infty}} .
$$

This implication substantiates that the system (1) is uniformly GFTS with respect to $\mathcal{A}=\left\{x \in \mathbb{R}^{n}:\|x\|_{\mathbf{d}} \leq A\right\}$ for small inputs for all $\nu_{\infty} \in \mathbb{R}$.

(2) If $\nu_{\infty}<0$, we use the same Lyapunov function $W(x)=\ln (1+V(x))$ as above, and we get

$$
\|x\|_{\mathbf{d}}>A \Longrightarrow\langle D W(x), f(x, \delta)\rangle \leq-\frac{a\|x\|_{\mathbf{d}}^{k+\nu_{\infty}}}{2(1+V(x))}+\tilde{c}\|\delta\|, \tilde{c}>0 .
$$

Then the system (1) is iISS with respect to $\mathcal{A}=\left\{x \in \mathbb{R}^{n}:\|x\|_{\mathbf{d}} \leq A\right\}$.

(3) If $\nu_{\infty}>0$, one gets the relation:

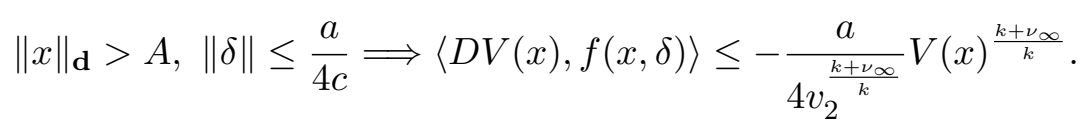

Recall that in this case $\frac{k+\nu_{\infty}}{k}>1$, denote $V_{0}=V(x(0))$, then we obtain the following estimate on the trajectory of $V(x(t))$ :

$$
\begin{aligned}
\|x\|_{\mathbf{d}}>A,\|\delta\| \leq \frac{a}{4 c} & \Longrightarrow V(x(t)) \leq \frac{1}{\left(V_{0}^{-\frac{\nu_{\infty}}{k}}+\frac{a v_{2}^{\frac{k+\nu_{\infty}}{k}}}{4} \frac{\nu_{\infty}}{k} t\right)^{\frac{k}{\nu_{\infty}}}}, \text { for all } V_{0}, \\
& \Longrightarrow V(x(t)) \leq \frac{1}{\left(\frac{a v_{2}^{\frac{k+\nu_{\infty}}{k}}}{4} \frac{\nu \infty}{k} t\right)^{\frac{k}{\nu_{\infty}}}} .
\end{aligned}
$$

Define the ball $\mathcal{B}=\left\{x \in \mathbb{R}^{n}: V(x) \leq v_{1} A^{k}\right\}$, which means that $\mathcal{B} \subset \mathcal{A}$. Obviously, for small inputs the time of convergence to the set $\mathcal{A}$ is upper bounded by $T=\frac{4 k}{a \nu_{\infty} v_{1}^{\frac{\nu_{\infty}}{k}} v_{2}^{\frac{k+\nu_{\infty}}{k}} A^{\nu_{\infty}}}$ and it is uniform (independent on initial conditions).

Example 3.5. Consider the system (1) with the vector field $f$ given by

$$
f\left((x, y),\left(\delta_{1}, \delta_{2}\right)\right)=\left(\begin{array}{ll}
0 & -y x^{2} \\
x^{3}+y^{4} & -y^{3}+x^{4}
\end{array}\right)\left[\left(\begin{array}{l}
1 \\
1
\end{array}\right)+\left(\begin{array}{l}
\delta_{1} \\
\delta_{2}
\end{array}\right)\right],
$$

for any $\delta \in \mathbb{R}^{m}$ it is homogeneous in 0 -limit with associated triple $\left(2, \mathbf{d}, f_{0}\right)$ with 
$\mathbf{d}(s)=\left(\begin{array}{ll}e^{s} & 0 \\ 0 & e^{s}\end{array}\right)$ and $f_{0}\left((x, y),\left(\delta_{1}, \delta_{2}\right)\right)=\left(\begin{array}{ll}0 & -y x^{2} \\ x^{3} & -y^{3}\end{array}\right)\left[\left(\begin{array}{l}1 \\ 1\end{array}\right)+\left(\begin{array}{l}\delta_{1} \\ \delta_{2}\end{array}\right)\right]$. For the homogeneous Lyapunov function $V(x, y)=\frac{1}{2} x^{2}+\frac{1}{2} y^{2}$, we get

$$
\left\langle D V(x, y), f_{0}((x, y),(0,0))\right\rangle=-y^{4} .
$$

It follows that the origin for the system $(\dot{x}, \dot{y})=f_{0}((x, y),(0,0))$ is GAS (using Lasalle invariance principle). Then, according to Theorem 3.3 the system $(\dot{x}, \dot{y})^{T}=$ $f\left((x, y),\left(\delta_{1}, \delta_{2}\right)\right)$ is uniformly GAS for small inputs.

Theorems 3.3 and 3.4 show that if a system has a homogeneous approximation, which satisfies the assumptions $\mathcal{H}_{1}, \mathcal{H}_{2}$ and $\mathcal{H}_{3}$, then it preserves some finite-time convergence even in the presence of exogenous inputs. These results will be used in Section 4.

\subsection{Robustness of finite-time stability of cascade}

Now we will evaluate robustness of two serially connected systems, which are homogeneous with different degrees of homogeneity:

$$
\begin{aligned}
& \dot{x}(t)=f(x(t), y(t)), \\
& \dot{y}(t)=g(y(t), \delta(t)),
\end{aligned}
$$

where $x(t) \in \mathbb{R}^{n}, y(t) \in \mathbb{R}^{m}$ are the state components and $\delta(t) \in \mathbb{R}^{p}$ represents an external input, $\delta \in \mathcal{L}_{\infty}$. Before studying the cascade (13), (14), we introduce the following lemma, which gives the conditions for forward existence of solutions for the system (13) with bounded inputs.

Lemma 3.6. If the system (13) satisfies the conditions $\mathcal{H}_{1}, \mathcal{H}_{2}$ with $\nu \leq 0$ and the input $y$ does not blow up in a finite time, then the solutions of the system (13) are well-defined for all $t \geq 0$.

Proof. By the imposed conditions $f(x, y)=f_{1}(x)+f_{2}(x) y$ with d-homogeneous vector fields $f_{1}$ and $f_{2}$ of degree $\nu$. Select any positive definite and continuously differentiable d-homogeneous function $V$ of degree $k>\max \{-\nu, 0\}$, then the relations (10) are satisfied. For any $x \in \mathbb{R}^{n}$ define $s=\ln \left(\|x\|_{\mathbf{d}}\right)$ and $\tilde{x} \in S$ such that $x=\mathbf{d}(s) \tilde{x}$, then by homogeneity of the function $f_{2}$ we derive the estimates:

$$
-\|y\| \sup _{\|\tilde{x}\|_{\mathbf{d}}=1}\left\|f_{2}(\tilde{x})^{T} D V(\tilde{x})\right\| \leq\left\langle f_{2}(\tilde{x})^{T} D V(\tilde{x}), y\right\rangle \leq\|y\| \sup _{\|\tilde{x}\|_{\mathbf{d}}=1}\left\|f_{2}(\tilde{x})^{T} D V(\tilde{x})\right\| .
$$


From this we obtain

$$
\begin{aligned}
\langle D V(x), f(x, y)\rangle & =\left\langle D V(x), f_{1}(x)\right\rangle+\left\langle D V(x), f_{2}(x) y\right\rangle \\
& =\left\langle D V(\mathbf{d}(s) \tilde{x}), f_{1}(\mathbf{d}(s) \tilde{x})\right\rangle+\left\langle D V(\mathbf{d}(s) \tilde{x}), f_{2}(\mathbf{d}(s) \tilde{x}) y\right\rangle \\
& =e^{(\nu+k) s}\left\langle D V(\tilde{x}), f_{1}(\tilde{x})\right\rangle+e^{(\nu+k) s}\left\langle D V(\tilde{x}), f_{2}(\tilde{x}) y\right\rangle \\
& =\|x\|_{\mathbf{d}}^{\nu+k}\left\langle D V(\tilde{x}), f_{1}(\tilde{x})\right\rangle+\|x\|_{\mathbf{d}}^{\nu+k}\left\langle f_{2}(\tilde{x})^{T} D V(\tilde{x}), y\right\rangle \\
& \leq\|x\|_{\mathbf{d}}^{\nu+k} \sup _{\|\tilde{x}\|_{\mathbf{d}}=1}\left[\left\langle D V(\tilde{x}), f_{1}(\tilde{x})\right\rangle\right] \\
& +\|x\|_{\mathbf{d}}^{\nu+k}\|y\|_{\|\tilde{x}\|_{\mathbf{d}}=1}\left\|f_{2}(\tilde{x})^{T} D V(\tilde{x})\right\| \\
& =\|x\|_{\mathbf{d}}^{\nu+k}(a+c\|y\|),
\end{aligned}
$$

and

$$
\begin{aligned}
\langle D V(x), f(x, y)\rangle= & \|x\|_{\mathbf{d}}^{\nu+k}\left(\left\langle D V(\tilde{x}), f_{1}(\tilde{x})\right\rangle+\left\langle f_{2}(\tilde{x})^{T} D V(\tilde{x}), y\right\rangle\right) \\
\geq & \|x\|_{\mathbf{d}}^{\nu+k}\left(\inf _{\|\tilde{x}\|_{\mathbf{d}}=1}\left[\left\langle D V(\tilde{x}), f_{1}(\tilde{x})\right\rangle\right]\right. \\
& \left.-\|y\| \sup _{\|\tilde{x}\|_{\mathbf{d}}=1}\left\|f_{2}(\tilde{x})^{T} D V(\tilde{x})\right\|\right) \\
= & \|x\|_{\mathbf{d}}^{\nu+k}\left(a^{\prime}-c\|y\|\right),
\end{aligned}
$$

where

$$
\begin{gathered}
a=\sup _{\|\tilde{x}\|_{\mathbf{d}}=1}\left[\left\langle D V(\tilde{x}), f_{1}(\tilde{x})\right\rangle\right], a^{\prime}=\inf _{\|\tilde{x}\|_{\mathbf{d}}=1}\left\langle D V(\tilde{x}), f_{1}(\tilde{x})\right\rangle \\
c=\sup _{\|\tilde{x}\|_{\mathbf{d}}=1}\left\|f_{2}(\tilde{x})^{T} D V(\tilde{x})\right\| .
\end{gathered}
$$

From (15) and (16), the derivative of $V$ for the system (13) satisfies the following relations:

$\|x(t)\|_{\mathbf{d}}^{k+\nu}\left(a^{\prime}-c\|y\|_{[0, t)}\right) \leq \dot{V}(t)=\langle D V(x(t)), f(x(t), y(t))\rangle \leq\|x(t)\|_{\mathbf{d}}^{k+\nu}\left(a+c\|y\|_{[0, t)}\right)$,

on the time interval $[0, t)$ for any finite $t \geq 0$ for which the solution $x(t)$ exists. Hence, if $\nu<0$ then an upper estimate on the behavior of $V(x(t))$ can be obtained:

$$
\dot{V}(x(\tau)) \leq\left(v_{1}^{-1} V(x(\tau))\right)^{\alpha}\left(a+c\|y\|_{[0, t)}\right)
$$

for all $\tau \in[0, t)$ and for $\alpha=\frac{k+\nu}{k}$, or equivalently

$$
V^{1-\alpha}(x(t)) \leq V^{1-\alpha}\left(x_{0}\right)+v_{1}^{-\alpha}(1-\alpha)\left(a+c\|y\|_{[0, t)}\right) t, x_{0} \in \mathbb{R}^{n} .
$$

Note that the right-hand side is bounded for all $t \geq 0$ provided that $\|y\|_{[0, t)}$ is finite (which is assumed in the conditions of the lemma). Therefore, $V(t)$ is well-defined and the solutions exists for any $t \geq 0$.

Lemma 3.6 shows that if the degree of homogeneity $\nu$ is negative, then the solutions of the system (13) can be extended to all $t \geq 0$ provided that the system (14) possesses 
the same property.

Remark 1. For the system (13) as in Lemma 3.6, if the degree of homogeneity $\nu$ is positive, then solutions of the system (13) may escape to infinity in a finite time for some inputs $y$.

The main result of this subsection given bellow characterizes the robustness properties of the interconnection $(13),(14)$ when the vector field $f$ of the system (13) satisfies the assumptions $\mathcal{H}_{1}, \mathcal{H}_{2}, \mathcal{H}_{3}$ and $y$ represents filtered by (14) bounded exogenous perturbation $\delta$.

\section{Theorem 3.7. If}

(a) the vector field $f$ satisfies the assumptions $\mathcal{H}_{1}, \mathcal{H}_{2}$ and $\mathcal{H}_{3}$ for $\nu<0$,

(b) the vector field $g$ is chosen such that

$$
e^{-\mu s} \boldsymbol{d}_{y}(-s) g\left(\boldsymbol{d}_{y}(s) y, \boldsymbol{d}_{\delta}(s) \delta\right)=g(y, \delta),
$$

$\forall s \in \mathbb{R}, \forall y \in \mathbb{R}^{m}, \forall \delta \in \mathbb{R}^{p}$ and some $\mu \in \mathbb{R}$, where diag $\left\{\boldsymbol{d}_{y}(s), \boldsymbol{d}_{\delta}(s)\right\}$ forms a generalized dilation,

(c) $\dot{y}=g(y, 0)$ is $G A S$.

Then, the system (13),(14) is

- strongly iISS for every $\mu \in \mathbb{R}$,

- if $\mu<0$ FTISS for small inputs.

Proof. Since (b) and (c) are verified, by using Theorem 3.2, we conclude that (14) is ISS for every $\mu \in \mathbb{R}$, and if $\mu<0$, then (14) is FTISS. According to (a) and using theorems 3.1 and 2.10, we deduce that the system (13), (14) is strongly iISS.

To prove global FTISS property for small inputs if $\mu<0$, select any $\left(x_{0}, y_{0}\right) \in$ $\mathbb{R}^{n} \times \mathbb{R}^{m}$, and denote by $z\left(t, z_{0}\right)=\left(x^{T}\left(t, x_{0}, y\right), y^{T}\left(t, y_{0}, \delta\right)\right)^{T}$ the common solution of the cascade for initial conditions $z_{0}=\left(x_{0}^{T}, y_{0}^{T}\right)^{T}$. Theorem 3.1 implies that for $\nu<0$ the system (13) is uniformly GFTS for small inputs, i.e., there exist $m>0$ and $\beta_{x} \in \mathcal{G} \mathcal{K} \mathcal{L}$ such that

$$
\begin{aligned}
\|y\|_{\left[0, T_{x}\left(x_{0}\right)\right) \leq m} & \Longrightarrow \\
& \Longrightarrow x\left(t, x_{0}, y\right) \| \leq \beta_{x}\left(\left\|x_{0}\right\|, t\right) \\
& \lim _{t \rightarrow T_{x}\left(x_{0}\right)}\left\|x\left(t, x_{0}, y\right)\right\|=0
\end{aligned}
$$

for all $x_{0} \in \mathbb{R}^{n}$, where $T_{x}: \mathbb{R}^{n} \rightarrow \mathbb{R}_{+}$is the settling-time function of the system (13). The system (14) is FTISS if $\mu<0$, i.e., there exist $\beta_{y} \in \mathcal{G K} \mathcal{L}$ and $\sigma \in \mathcal{K}$ such that

$$
\left\|y\left(t, y_{0}, \delta\right)\right\| \leq \beta_{y}\left(\left\|y_{0}\right\|, t\right)+\sigma\left(\|\delta\|_{[0, t)}\right) \Longrightarrow \sup _{t \geq T_{y}\left(y_{0}\right)}\left\|y\left(t, y_{0}, \delta\right)\right\| \leq \sigma\left(\|\delta\|_{\infty}\right)
$$

for all $y_{0} \in \mathbb{R}^{m}$ and $\delta \in \mathcal{L}_{\infty}$, where $T_{y}: \mathbb{R}^{n} \rightarrow \mathbb{R}_{+}$is the settling-time function of the system (14).

Assume that $\|\delta\|_{\infty} \leq \sigma^{-1}\left(\frac{m}{2}\right)$.

First case: If $\beta_{y}\left(\left\|y_{0}\right\|, 0\right) \leq \frac{m}{2}$, this implies that $\left\|y\left(t, y_{0}, \delta\right)\right\| \leq m, \forall t \geq 0$, then

$$
\lim _{t \rightarrow T_{x}\left(x_{0}\right)}\left\|x\left(t, x_{0}, y\right)\right\|=0 .
$$


Consequently,

$$
\begin{aligned}
\lim _{t \rightarrow \max \left\{T_{x}\left(x_{0}\right), T_{y}\left(y_{0}\right)\right\}}\left\|z\left(t, z_{0}\right)\right\| & \leq \lim _{t \rightarrow \max \left\{T_{x}\left(x_{0}\right), T_{y}\left(y_{0}\right)\right\}}\left(\left\|x\left(t, x_{0}, y\right)\right\|+\left\|y\left(t, y_{0}, \delta\right)\right\|\right) \\
& \leq \sigma\left(\|\delta\|_{\infty}\right) .
\end{aligned}
$$

Second case: Let $\beta_{y}\left(\left\|y_{0}\right\|, 0\right)>\frac{m}{2}$, since $\beta_{y} \in \mathcal{G K} \mathcal{L}$ there exists $T_{y_{0}}>0$ such that

$$
t \geq T_{y_{0}} \Longrightarrow \beta_{y}\left(\left\|y_{0}\right\|, t\right) \leq \frac{m}{2}
$$

which implies that

$$
\|y(t)\| \leq m, \forall t \geq T_{y_{0}},
$$

and, consequently, $x\left(t, x_{0}, y\right)$ will converges to zero provided that it is well defined for $t \in\left[0, T_{y_{0}}\right]$. Using the result of Lemma 3.6 we substantiate that the value $x\left(T_{y_{0}}, x_{0}, y\right)$ is finite for any $x_{0} \in \mathbb{R}^{n}$ (see the estimate obtained in the proof), then

$$
\left\|x\left(t, x\left(T_{y_{0}}\right), y\right)\right\| \leq \beta_{x}\left(\left\|x\left(T_{y_{0}}\right)\right\|, t-T_{y_{0}}\right) \Longrightarrow \lim _{t \rightarrow T_{x}\left(x\left(T_{y_{0}}\right)\right)+T_{y_{0}}}\left\|x\left(t, x\left(T_{y_{0}}\right), y\right)\right\|=0 .
$$

Which leads to

$$
\begin{aligned}
& \lim _{\leq} \lim _{t \rightarrow \max \left\{T_{x}\left(x\left(T_{y_{0}}\right)\right)+T_{y_{0}}, T_{y}\left(y_{0}\right)\right\}} \lim _{t \rightarrow \max \left\{T_{x}\left(x\left(T_{y_{0}}\right)\right)+T_{y_{0}}, T_{y}\left(y_{0}\right)\right\}}\left(\left\|x\left(t, x_{0}, y\right)\right\|+\left\|y\left(t, y_{0}, \delta\right)\right\|\right) \\
& \leq \sigma\left(\|\delta\|_{\infty}\right),
\end{aligned}
$$

and the finite-time convergence is proven for small inputs. Applying the same arguments the global boundedness of all trajectories can be established. Then we deduce that the system (13),(14) is FTISS for small inputs for the case $\nu<0$ and $\mu<0$.

In the above theorem we also have shown that in the latter case the system (13),(14) is uniformly globally FTS for small inputs.

The following corollary extends the above stability result to the interconnected system $(13),(14)$ when the vector field $f$ of the system (13) has a homogeneous approximation.

Corollary 3.8. Assume that conditions $(b)$ and $(c)$ of Theorem 3.7 are satisfied, while the condition (a) is replaced with

- $f(\cdot, y)$ is homogeneous in 0-limit (resp., $\infty$-limit) with associated triple $\left(\nu_{0}, \boldsymbol{d}, f_{0}(\cdot, y)\right)$ (resp., $\left.\left(\nu_{\infty}, \boldsymbol{d}, f_{\infty}(\cdot, y)\right)\right)$, where $\boldsymbol{d}$ is a generalized linear dilation and $\nu_{0}<0$ (resp., $\left.\nu_{\infty}<0\right)$, and $f_{0}$ (resp., $f_{\infty}$ ) satisfies the assumptions $\mathcal{H}_{1}, \mathcal{H}_{2}$ and $\mathcal{H}_{3}$.

Then, for $\mu<0$ the interconnected system (13),(14) is locally FTISS (resp., is FTISS with respect to $\mathcal{B}=\left\{(x, y) \in \mathbb{R}^{n} \times \mathbb{R}^{m}:\|x\|_{\boldsymbol{d}_{x}} \leq A, y=0\right\}$ for some $A>0$ ) for small inputs.

Proof. By using the conditions of Corollary 3.8 and Theorem 3.7 we deduce that the system (14) is ISS. If $\nu_{0}<0$, from Theorem 3.3, the system (13) is uniformly FTS for 
small inputs. By using the same method as in the Step II of the proof of Theorem 3.7 , we prove that if $\mu<0$, the interconnected system $(13),(14)$ is locally FTISS for small inputs.

Theorem 3.4 implies that, if we choose $A>0$ sufficiently big, for all $\left\|x_{0}\right\|>A$, if $\nu_{\infty}<0$, the system (13) is uniformly GFTS for small inputs to the set $\mathcal{A}=$ $\left\{x \in \mathbb{R}^{n}:\|x\|_{\mathbf{d}_{x}} \leq A\right\}$. Using the same method as in the proof of Theorem 3.7 one deduces that the set $\mathcal{B}$ is FTISS for small inputs for the interconnected system (13),(14) when $\mu<0$.

Remark 2. Let instead of (14), we have

$$
\dot{y}=g(y),
$$

where $g$ is $\mathbf{d}_{y}$-homogeneous function with degree of homogeneity $\mu$ and the system (17) is GAS. Then if $\nu<0$, the dynamical system (13),(17) is GAS for all $\mu \in \mathbb{R}$. If $\nu<0$ and $\mu<0$ in the conditions of Theorem 3.7, then the system (13),(17) is GFTS.

In the next section, to illustrate the utility of these results we are going to analyze the robustness of a finite-time observer with respect to time variations of its gains.

\section{Application}

In (Perruquetti et al., 2008) a finite-time observer is proposed for a nonlinear system of the form:

$$
\begin{aligned}
\dot{z} & =A z+f(\psi, u), \\
\psi & =C z,
\end{aligned}
$$

where $z \in \mathbb{R}^{n}$ is the state, $u \in \mathbb{R}^{m}$ in a known input and $\psi \in \mathbb{R}$ is the measured output, and the matrices

$$
A=\left(\begin{array}{ccccc}
0 & 1 & 0 & \cdots & 0 \\
0 & 0 & 1 & \cdots & 0 \\
\vdots & \vdots & \ddots & \ddots & \vdots \\
0 & 0 & \ldots & \ddots & 1 \\
0 & 0 & \ldots & \ldots & 0
\end{array}\right), C=(1,0, \cdots, 0)
$$

are in a canonical representation. An observer for this system is designed as

$$
\dot{\hat{z}}=A \hat{z}+f(\psi, u)+\left(\begin{array}{c}
k_{1}\left|z_{1}-\hat{z}_{1}\right|^{\alpha_{1}} \operatorname{sign}\left(z_{1}-\hat{z}_{1}\right) \\
k_{2}\left|z_{1}-\hat{z}_{1}\right|^{\alpha_{2}} \operatorname{sign}\left(z_{1}-\hat{z}_{1}\right) \\
\vdots \\
k_{n}\left|z_{1}-\hat{z}_{1}\right|^{\alpha_{n}} \operatorname{sign}\left(z_{1}-\hat{z}_{1}\right)
\end{array}\right) \text {, }
$$

where $\hat{z} \in \mathbb{R}^{n}$ is an estimate of $z, \alpha_{i}$ is a constant power and $k_{i}$ is a constant observer gain for $1 \leq i \leq n$. The dynamics of the estimation error $e=z-\hat{z}$ can be written as 
follows:

$$
\begin{cases}\dot{e}_{1} & =e_{2}-k_{1}\left|e_{1}\right|^{\alpha_{1}} \operatorname{sign}\left(e_{1}\right) \\ \dot{e}_{2} & =e_{3}-k_{2}\left|e_{1}\right|^{\alpha_{2}} \operatorname{sign}\left(e_{1}\right) \\ \vdots & \\ \dot{e}_{n-1} & =e_{n-1}-k_{n-1}\left|e_{1}\right|^{\alpha_{n-1}} \operatorname{sign}\left(e_{1}\right) \\ \dot{e}_{n} & =-k_{n}\left|e_{1}\right|^{\alpha_{n}} \operatorname{sign}\left(e_{1}\right)\end{cases}
$$

To guarantee FTS of the system (18), the homogeneity framework is used (Perruquetti et al., 2008) resulting in the choice $\alpha_{i}=1+i \alpha, 1 \leq i \leq n$, where $\alpha>-\frac{1}{n}$ is a tuning parameter that represents the homogeneity degree of the system, for the dilation

$$
\mathbf{d}_{x}(s)=\operatorname{diag}\left[e^{s}, e^{s(1+\alpha)}, \ldots, e^{s(1+(n-1) \alpha)}\right] .
$$

The gains $k_{i}, 1 \leq i \leq n$ should be selected to form a Hurwitz polynomial.

\subsection{Homogeneous affine nonlinear dynamical system}

Our goal is to study the same problem with multiplicative disturbances introduced as follows:

$$
\dot{\hat{z}}=A \hat{z}+f(\psi, u)+\left(\begin{array}{c}
\left(k_{1}+y_{1}\right)\left|z_{1}-\hat{z}_{1}\right|^{1+\alpha} \operatorname{sign}\left(z_{1}-\hat{z}_{1}\right) \\
\left(k_{2}+y_{2}\right)\left|z_{1}-\hat{z}_{1}\right|^{1+2 \alpha} \operatorname{sign}\left(z_{1}-\hat{z}_{1}\right) \\
\vdots \\
\left(k_{n}+y_{n}\right)\left|z_{1}-\hat{z}_{1}\right|^{1+n \alpha} \operatorname{sign}\left(z_{1}-\hat{z}_{1}\right)
\end{array}\right)
$$

where all symbols keep their meaning, and $y_{i} \in \mathbb{R}, 1 \leq i \leq n$ represent variations of the gains $k_{i}, 1 \leq i \leq n$ occurred due to an additional on-line tuning (via adaptation algorithms) or due to an auxiliary measurement information (usually the values of $k_{i}$ are related with the amplitude of uncertainty to compensate).

It is straightforward to verify that the error dynamics for (19) satisfies the hypotheses $\mathcal{H}_{1}$ and $\mathcal{H}_{2}$ for $f(e, y)=f_{1}(e)+f_{2}(e) y$ with

$$
f_{1}(e)=\left(\begin{array}{c}
-k_{1}\left|e_{1}\right|^{1+\alpha} \operatorname{sign}\left(e_{1}\right)+e_{2} \\
-k_{2}\left|e_{1}\right|^{1+2 \alpha^{2}} \operatorname{sign}\left(e_{1}\right)+e_{3} \\
\vdots \\
-k_{n}\left|e_{1}\right|^{1+n \alpha} \operatorname{sign}\left(e_{1}\right)
\end{array}\right)
$$

and

$$
f_{2}(e)=-\operatorname{diag}\left[\left|e_{1}\right|^{1+\alpha} \operatorname{sign}\left(e_{1}\right), \ldots,\left|e_{1}\right|^{1+n \alpha} \operatorname{sign}\left(e_{1}\right)\right],
$$

and the functions $f_{1}$ and $f_{2}$ are $\mathbf{d}_{x}$-homogeneous with the same degree of homogeneity $\alpha$. Using the method proposed in (Perruquetti et al., 2008) it is possible to show that Assumption $\mathcal{H}_{3}$ is also verified for $\alpha$ sufficiently close to zero (of both signs). Therefore, Theorem 3.1 allows us to conclude that the estimation error dynamics for the observer (19) is uniformly GAS for small inputs for any $\alpha>-\frac{1}{n}$ and it is strongly finite-time iISS and uniformly GFTS for small inputs if $\alpha \in\left(-\frac{1}{n}, 0\right)$. 


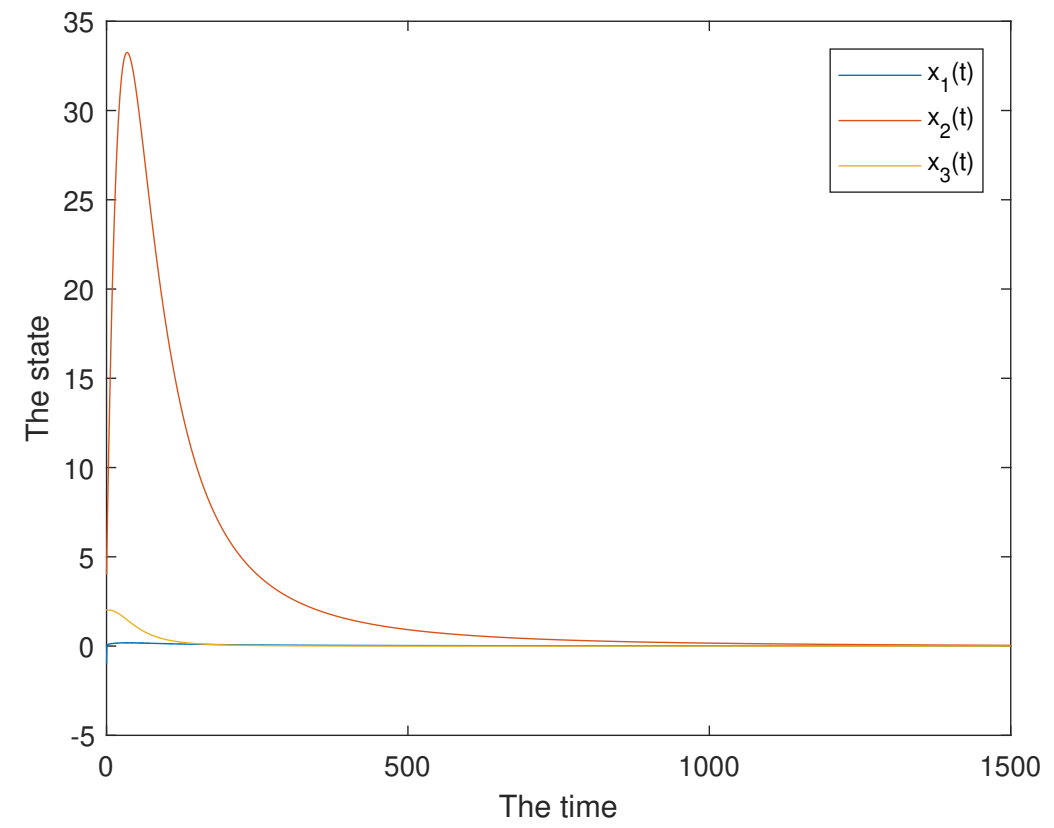

Figure 1. The solutions of the system (19) with the initial condition $\left(x_{1,0}, x_{2,0}, x_{3,0}\right)=(-1,2,1), y(t)=$ $\left(\cos (t), \frac{t}{1+t^{2}}+2,200 \frac{t}{1+t^{2}}+\cos \left(1+t^{2}\right)\right)^{T}$ and $\alpha=1$.

Let $n=3$ and select $k_{1}=1000, k_{2}=240, k_{3}=24$, then figures 1 and 2 show the uniform GAS and GFTS of the state of the system (19) for different degrees $\alpha$ and sufficiently small inputs $y$.

\subsection{Interconnected system}

In order to illustrate the results of Theorem 3.7 we will consider also the system (19) in a cascade with

$$
\dot{y}=g(y, \delta),
$$

which may represent the adaptation algorithm influenced by a measurement noise or a disturbance $\delta \in \mathbb{R}^{p}$ (a stable filter).

For simulation with $n=3$ let

$$
g(y, \delta)=\left(\begin{array}{c}
y_{2}^{\frac{1}{3}}-\left|y_{1}\right|^{\frac{1}{2}} \operatorname{sign}\left(y_{1}\right) \\
y_{3}-y_{1}-y_{2}^{\frac{2}{3}} \operatorname{sign}\left(y_{2}\right) \\
-y_{2}^{\frac{1}{3}}-\left|y_{3}\right|^{\frac{1}{2}} \operatorname{sign}\left(y_{3}\right)+\delta
\end{array}\right),
$$

where $g: \mathbb{R}^{3} \times \mathbb{R} \longmapsto \mathbb{R}^{3}$ is continuous and d-homogeneous with degree of homogeneity $\mu=-\frac{1}{3}, \mathbf{d}(s)=\left(\begin{array}{llll}e^{\frac{2}{3} s} & 0 & 0 & 0 \\ 0 & e^{s} & 0 & 0 \\ 0 & 0 & e^{\frac{2}{3} s} & 0 \\ 0 & 0 & 0 & e^{\frac{1}{3} s}\end{array}\right)$ and the system $\dot{y}(t)=g(y(t), 0)$ is GAS. 


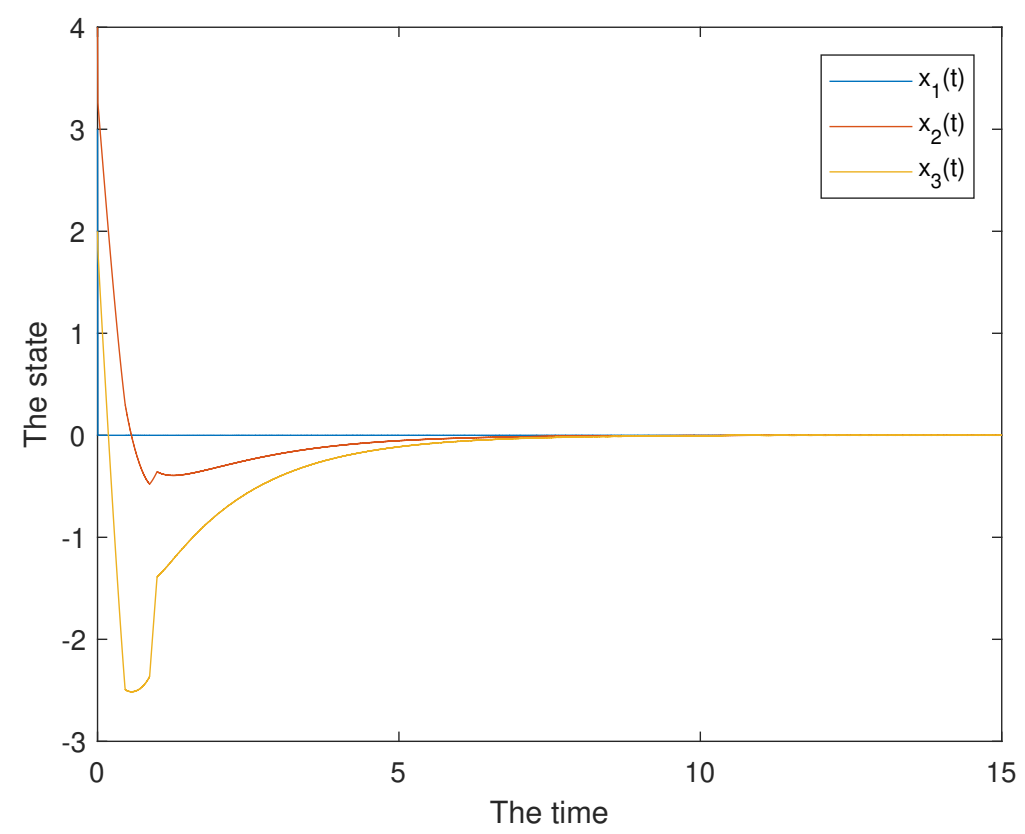

Figure 2. The solutions of the system (19) with the initial condition $\left(x_{1,0}, x_{2,0}, x_{3,0}\right)=(-1,2,1), y(t)=$ $\left(\cos (t), \frac{t}{1+t^{2}}+2,200 \frac{t}{1+t^{2}}+\cos \left(1+t^{2}\right)\right)^{T}$ and $\alpha=0.7$.

Then, if $\frac{1}{n}<\alpha<0$, Theorem 3.7 implies that the systems (19), (20) in cascade are finite-time ISS for small inputs and finite-time strongly iISS. From Figure 3 we see that the state is bounded when inputs are bounded.

\section{Conclusion}

In this paper, we studied the robustness features of finite-time stable homogeneous dynamical systems with respect to additive perturbations. Some extensions are established for the systems admitting homogeneous approximations at the origin and at infinity. Influence on robustness of additional dynamics in the input channel is also investigated. The efficiency and practicality of the obtained conditions are demonstrated by considering a homogeneous observer with the gains dependent on functions of time, i.e., on additional measured information or adaptive tuning. Simulation results and academic examples are included for illustration.

\section{References}

Andrieu, V., Praly, L., \& Astolfi, A. (2008). Homogeneous approximation, recursive observer design, and output feedback. SIAM Journal on Control and Optimization, 47(4), 18141850.

Angeli, D., Sontag, E. D., \& Wang, Y. (2000). A characterization of integral input-to-state stability. IEEE Transactions on Automatic Control, 45(6), 1082-1097.

Arcak, M., \& Kokotović, P. (2001). Nonlinear observers: a circle criterion design and robustness analysis. Automatica, 37(12), 1923-1930. 


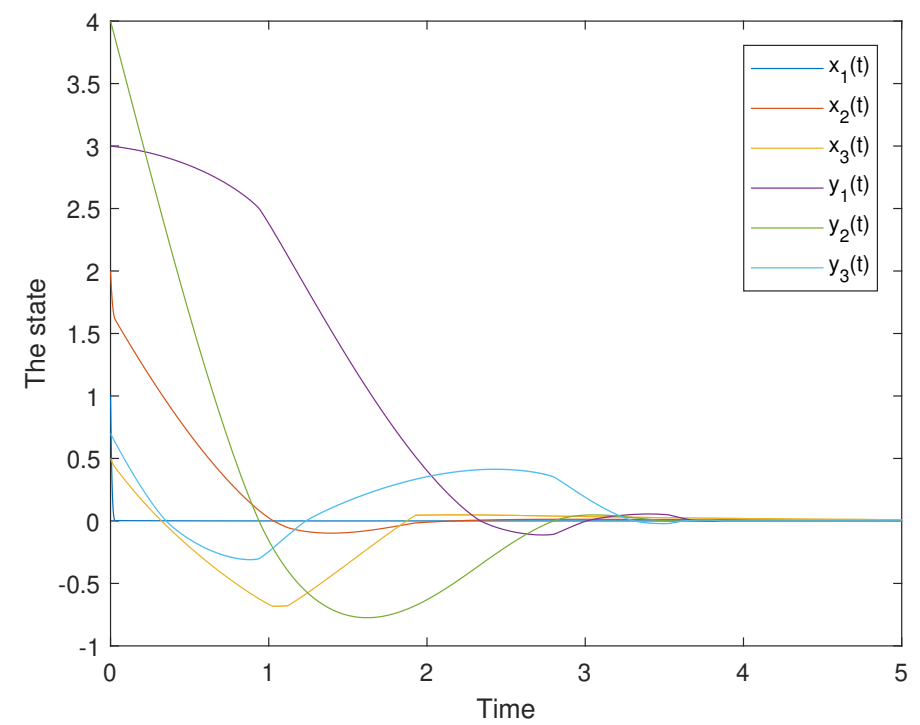

Figure 3. The solutions of the system (19) with the initial condition $\left(x_{1,0}, x_{2,0}, x_{3,0}, y_{1,0}, y_{2,0}, y_{3,0}\right)=$ $(1,2,0.5,3,4,0.7), \delta(t)=\cos (t)$ and $\alpha=0.7$.

Bacciotti, A., \& Rosier, L. (2006). Liapunov functions and stability in control theory. Springer Science \& Business Media.

Bernuau, E., Efimov, D., Perruquetti, W., \& Polyakov, A. (2013). On an extension of homogeneity notion for differential inclusions. In Control conference ecc, 2013 european (pp. 2204-2209).

Bernuau, E., Efimov, D., Perruquetti, W., \& Polyakov, A. (2014). On homogeneity and its application in sliding mode control. Journal of the Franklin Institute, 351(4), 1866-1901.

Bernuau, E., Polyakov, A., Efimov, D., \& Perruquetti, W. (2013a, 02). Robustness of finitetime stability property for sliding modes. , 391-396.

Bernuau, E., Polyakov, A., Efimov, D., \& Perruquetti, W. (2013b). Verification of ISS, iISS and IOSS properties applying weighted homogeneity. Systems \& Control Letters, 62 (12), $1159-1167$.

Bhat, S. P., \& Bernstein, D. S. (2000). Finite-time stability of continuous autonomous systems. SIAM Journal on Control and Optimization, 38(3), 751-766.

Bhat, S. P., \& Bernstein, D. S. (2005). Geometric homogeneity with applications to finite-time stability. Mathematics of Control, Signals and Systems, 17(2), 101-127.

Braidiz, Y., Efimov, D., Polyakov, A., \& Perruquetti, W. (2019, June). Robust finite-time stability of homogeneous systems with respect to multiplicative disturbances. In 2019 18th European Control Conference (ECC) (p. 668-673).

Chaillet, A., Angeli, D., \& Ito, H. (2014a). Combining iISS and ISS with respect to small inputs: the strong iiss property. IEEE Transactions on Automatic Control, 59(9), 25182524.

Chaillet, A., Angeli, D., \& Ito, H. (2014b). Strong iISS is preserved under cascade interconnection. Automatica, 50(9), 2424-2427.

Coron, J.-M. (2007). Control and nonlinearity (No. 136). American Mathematical Soc.

Efimov, D., \& Perruquetti, W. (2010). Oscillations conditions in homogenous systems. In 8th ifac symposium on nonlinear control systems (pp. 1379-1384).

Efimov, D., \& Perruquetti, W. (2016). On conditions of oscillations and multi-homogeneity. Mathematics of Control, Signals, and Systems, 28(1), 3.

Freeman, R., \& Kokotovic, P. V. (2008). Robust nonlinear control design: state-space and lyapunov techniques. Springer Science and Business Media. 
Hahn, W. (1967). Stability of motion (Vol. 138). Springer.

Hahn, W., Hosenthien, H. H., \& Lehnigk, H. (1963). Theory and application of Lyapunov's direct method. Prentice-Hall Englewood Cliffs.

Hong, Y., Jiang, Z.-P., \& Feng, G. (2008). Finite-time input-to-state stability and applications to finite-time control. IFAC Proceedings Volumes, 41(2), 2466-2471.

Jiang, Z.-P., Teel, A. R., \& Praly, L. (1994). Small-gain theorem for ISS systems and applications. Mathematics of Control, Signals and Systems, 7(2), 95-120.

Korobov, V. (1979). A general approach to synthesis problem. Doklady Academii Nauk SSSR, 248, 1051-1063.

Moulay, E., \& Perruquetti, W. (2006). Finite time stability and stabilization of a class of continuous systems. Journal of Mathematical Analysis and Applications, 323(2), 14301443.

Perruquetti, W., Floquet, T., \& Moulay, E. (2008). Finite-time observers: application to secure communication. IEEE Transactions on Automatic Control, 53(1), 356-360.

Polyakov, A. (2018). Sliding mode control design using canonical homogeneous norm. International Journal of Robust and Nonlinear Control.

Polyakov, A., Coron, J.-M., \& Rosier, L. (2018). On homogeneous finite-time control for linear evolution equation in Hilbert space. IEEE Transactions on Automatic Control.

Polyakov, A., Efimov, D., Fridman, E., \& Perruquetti, W. (2016). On homogeneous distributed parameter systems. IEEE Transactions on Automatic Control, 61(11), 3657-3662.

Rosier, L. (1992). Homogeneous Lyapunov function for homogeneous continuous vector field. Systems \& Control Letters, 19(6), 467-473.

Roxin, E. (1966). On finite stability in control systems. Rendiconti del Circolo Matematico di Palermo, 15(3), 273-283.

Sontag, E. D. (1989). Smooth stabilization implies coprime factorization. IEEE Transactions on Automatic Control, $34(4), 435-443$.

Sontag, E. D. (1998). Comments on integral variants of ISS. Systems and Control Letters, $34(1-2), 93-100$.

Sontag, E. D., \& Wang, Y. (1995). On characterizations of the input-to-state stability property. Systems and Control Letters, $24(5), 351-359$.

Zubov, V. (1958 (in Russian)). On systems of ordinary differential equations with generalized homogenous right-hand sides. Izvestia vuzov. Mathematica., 1, 80-88. 